\title{
The Electrical Conductivity of Weakly Ionized Plasma \\ Containing Dust Particles
}

\author{
Hui $\mathrm{Li}^{1,2}$, Jian $\mathrm{Wu}^{2}$, Chengxun $\quad$ Yuan $^{1}$ and Zhongxiang Zhou ${ }^{1}$ \\ 1. Department of physics, Harbin Institute of Technology, Harbin, China \\ 2. China Research Institute of Radio wave Propagation (CRIRP), Beijing, China
}

The effect of charged dust particle on the electrical conductivity of weakly ionized dusty plasma is investigated. It is shown that the additional collision provided by charged dust particles can significantly alter the electrical conductivity of electron-ion plasma. The numerical results indicated that these effects are mainly determined by dust radius, density as well as the charge numbers on dust surface. The obtained results will support an enhanced understanding of the electromagnetic wave propagation processes in dusty plasma.

\section{Introduction}

Plasma containing a large numbers of various-sized dust particles, from a few nanometers to tens of micrometers, has attracted substantial interest within the investigations. This interest can be attributed to their importance for a number of applications ranging from astrophysics to space physics, as well as some man-made environments. Such plasmas are referred to as dusty plasmas or complex plasmas. The dust particles immersed in plasma can be charged by plasma currents and other effects, depending on dust size and ambient plasma conditions. Since the presence of highly charged and massive dust particle in plasma exhibit completely different dynamical behaviors with the electron and ion, as well as the interaction with the plasma leads to the appearance of new physical phenomena and to modification of physics of fundamental plasma. The physics of dusty plasma have prompted a substantial numbers of investigations over the last couple of decades ${ }^{1-10}$.

The electrical conductivity is an essential parameter utilized to describe the electromagnetic properties of plasma. The electrical conductivity of dusty plasma should be substantially different from electron-ion plasma. The main reason is that the dust particle in plasma are highly charged (usually can reach the value $10^{3}$ to $10^{4}$ ) and 
their size are many orders of magnitude than ions, the Coulomb collision provided by charged dust particle becomes significant and may compete with the other collisions process, even in weakly ionized plasma conditions. This collision associated with the charged dust particle can change the plasma density distribution, which in turn affects the conductivity of plasma. However, the effect of this collision process on the conductivity of plasma has not been considered as yet.

The purpose of this paper is to study the conductivity of dusty plasma by taking into account the collision process between the electron and charged dust particles. In the next section, we will detail analysis of the collision process involving charged dust particle. Then the variation of conductivity with respect to electromagnetic wave frequency for different dust radius and density is calculated. And the effect of dust size distribution on the conductivity is also discussed. Finally, we summarize our results and draw some conclusion.

\section{The model equations}

We assume that spherical dust particles immersed in plasma. The dust charge is governed by the charging equation ${ }^{11}$.

$$
\frac{d Q}{d t}=\sum I_{a}
$$

Where $I_{a=e, i}$ is the electron and ion current and $Q$ is the dust charge. For negatively charged dust, the current are given by:

$$
\left\{\begin{array}{l}
I_{e}=-4 \pi r_{d}^{2} n_{e} e\left(\frac{k_{B} T_{e}}{2 \pi m_{e}}\right)^{1 / 2} \exp \left(\frac{e \phi_{d}}{k_{B} T_{e}}\right) \\
I_{i}=4 \pi r_{d}^{2} n_{i} e\left(\frac{k_{B} T_{i}}{2 \pi m_{i}}\right)^{1 / 2}\left(1-\frac{e \phi_{d}}{k_{B} T_{i}}\right)
\end{array}\right.
$$

Where $r_{d}$ is the dust particle radius, $m_{e}$ and $m_{i}$ are the electron and ion mass, $n_{e}$ and $n_{i}$ are the electron and ion density, $T_{e}$ and $T_{i}$ are the electron and ion temperature. Here $\phi_{d}$ is the surface potential of the particle. We introduce some dimensionless variables, $p_{e}=n_{d} z_{d} / n_{e}$ stands for the ratio of the charge density carried by the dust particle to the electron number density, and $z=z_{d} e^{2} / r_{d} T_{e}$ is the 
dimensionless surface potential of the dust, $\tau=T_{i} / T_{e}$, and $\mu=m_{i} / m_{e}$. Here $n_{d}$ is the dust density and $Z_{d}$ represents charge numbers. In the charging equilibrium state $I_{i}+I_{e}=0$, and $\phi_{d}=-\left|Z_{d}\right| e / r_{d}$. Then we can obtain,

$$
\exp (-z)=\frac{\left(1+p_{e}\right)(\tau+z)}{\sqrt{\tau \mu}}
$$

Using equation (3), we can numerical calculation the dimensionless surface potential of dust $\mathrm{z}$.

Considering that the velocity of electrons is substantially faster than that of the ions, thus only contribution of collisions between electrons and other particles are considered in the present study. The expression of conductivity can be obtained by the Boltzmann kinetic equation. The corresponding kinetic equations for the electron taking into account collisions process with distribution $f_{e}(r, v, t)$ satisfies the following form ${ }^{12}$

$$
\frac{\partial f_{e}}{\partial t}+v \cdot \frac{\partial f_{e}}{\partial r}+\frac{e}{m_{e}} E \cdot \frac{\partial f_{e}}{\partial v}=\left.\frac{\partial f_{e}}{\partial t}\right|_{\text {coll }}
$$

Where $E$ represents the electric field, and $\partial f_{e} /\left.\partial t\right|_{\text {coll }}$ describes the rate of change for the distribution function due to various collision processes. In order to solve the equation (4), we introduced the perturbation distribution function $\delta f_{e}(r, v, t)$, thus the plasma distribution function is written as:

$$
f_{e}(r, v, t)=f_{e 0}(v)+\delta f_{e}(r, v, t),\left|\delta f_{e}\right|<<\left|f_{e 0}\right|
$$

Where $f_{e 0}$ is the unperturbed distribution function. The Maxwellian distribution of the electron density is expressed as:

$$
f_{e 0}=N_{e}\left(\frac{m_{e}}{2 \pi k_{B} T}\right)^{3 / 2} \exp \left(-\frac{m_{e} v^{2}}{2 k_{B} T}\right)
$$

To investigate a small perturbation $\delta f_{e}$ for the equilibrium, we linearize the equation (4) and using the BGK model approximate. Thus the solution of this linear kinetic equation for plane wave $\delta f_{e} \sim \exp (-i \omega \cdot t+i k \cdot r)$ can be written as 


$$
\delta f_{e}=-\frac{i}{\omega-k v+i v_{e f f}} \cdot \frac{e}{m_{e}} E \frac{\partial f_{e 0}}{\partial v}
$$

Here $v_{\text {eff }}$ is the effective collision frequency. Due to the perturbation, induced currents emerge in the plasma. The current density $\mathbf{j}$ depends on the perturbation distribution function

$$
\mathbf{j}=\int e \cdot v \cdot \delta f_{e} d v
$$

According to the Ohm law $\mathbf{j}=\sigma \cdot E$, for $\omega: v_{\text {eff }}>>k v$ high-frequency limit, the complex conductivity can be expressed as:

$$
\sigma_{\text {complex }}=\frac{\varepsilon_{0} \omega_{p e}^{2} v_{\text {eff }}}{\omega^{2}+v_{\text {eff }}^{2}}-i \omega \frac{\varepsilon_{0} \omega_{p e}^{2}}{\omega^{2}+v_{\text {eff }}^{2}}
$$

Where $\omega_{p e}=\sqrt{e^{2} N_{e} / \varepsilon_{0} m_{e}}$ is plasma frequency and $v_{e f f}=v_{e d}+v_{e n}$ is total collision rate involving the electron-neutral and electron-dust collision. Electron collisions with neutrals can be described by the familiar equation $v_{e n}=n_{n} v_{T e} \sigma_{e n}$, where $\sigma_{e n}$ is the collision cross section and $n_{n}$ is the neutral number density and $v_{T e}=\sqrt{k_{B} T_{e} / m_{e}}$ is the thermal velocity of electrons. Due to the dust particle in plasma are charged, the collisions of electrons with charged dust particles are more complex and should be divided into two processes, including direct collisions with charged dust particles $v_{e d}^{c h}$ and Coulomb scattering by the charged dust particles $v_{e d}^{c o}$. The first process is often referred to the charging process and can be described as:

$$
v_{e d}^{c h}=2 \sqrt{2 \pi} n_{d} v_{T e} r_{d}^{2} e^{-z}
$$

For the Coulomb scattering, the standard Coulomb scattering approach may be applied to describe electron Coulomb scattering by the charged dust particle. The detailed investigation of the Coulomb scattering cross section is given by Khrapak (see, Refs [13-15]) and the collision frequency for the Maxwellian particle distribution can be written in the form

$$
v_{e d}^{c o}=(8 \sqrt{2 \pi} / 3) n_{d} v_{T e} r_{d}^{2} z^{2} \Lambda_{e d}
$$


Where $\Lambda_{e d}$ is the Coulomb logarithm for electron-dust collisions, which can calculate approximately by $\Lambda_{e d} ; 2 \ln (2 L / z)$ and $L=\lambda_{D} / r_{d}$ is the ratio of screening length to dust radius. It can be seen from the Eq. (10) and (11) that the relative importance of Coulomb scattering and charging collisions are strongly depends on the factor $z$, when $z \geq 1$, the charging collision contribution becomes exponentially small, but the contribution from Coulomb scattering grows with the squared of $z$. What is worth mentioning is that the Eq. (11) has been obtained by assuming a Yukawa potential for the interaction between charged particle in dusty plasma and neglect any type of multiple collisions. The collisions with neutrals may deviations of particle potential from the Yukawa form, which in turn affect the Coulomb scattering process. Nevertheless, in many cases this simple model does provide reasonable predictions and can be considered as the basis for more sophisticated model.

\section{Numerical results and discussions}

The exhaust plume from solid rocket motors can be characterized as weakly ionized dusty plasma, which contains a large numbers of aluminum oxide particles $\left(\mathrm{Al}_{2} \mathrm{O}_{3}\right)^{16,17}$. Plasma and dust parameters of the rocket exhaust measured by experiments (Ref. $[17,18]$ ) indicate that size of the dust particles is typically $0.1 \mu \mathrm{m}$ to $10 \mu \mathrm{m}$, dust density is approximately $10^{10} \mathrm{~m}^{-3}$ to $10^{15} \mathrm{~m}^{-3}$, the ion density $n_{i}$ is often $10^{16} \mathrm{~m}^{-3}$ to $10^{18} \mathrm{~m}^{-3}$, and neutral density is $10^{20} \mathrm{~m}^{-3}$ to $10^{24} \mathrm{~m}^{-3}$. As a reference case, we assume $n_{d}=10^{12} \mathrm{~m}^{-3}, n_{i}=10^{17} \mathrm{~m}^{-3}$ and the temperature $T=2000 \mathrm{~K}$ in the model to calculate the conductivity of dusty plasma.

Fig. 1 shows the variations of conductivity $\sigma$ with respect to electromagnetic wave frequency for different dust size $r_{d}=1.0 \mu \mathrm{m}$ and $r_{d}=5.0 \mu \mathrm{m}$, respectively and the case without dust particles is also given for comparison. It can be concludes from Fig.1 that the presence of charged dust particles can alter the conductivity of electron-ion plasma. For the low frequency range, the additional collision provided by charged dust particle can reduce the conductivity. On the contrary, this additional 
collision increases the conductivity, when the electromagnetic wave frequency is close to and greater than the total collision frequency. We can also see that these change trends is enhanced with the increase of dust radius. This can be physically interpreted as due to the larger dust particle not only have more charges on the surface, but also have lager scattering cross section, which can increase the electron-dust collision rate.

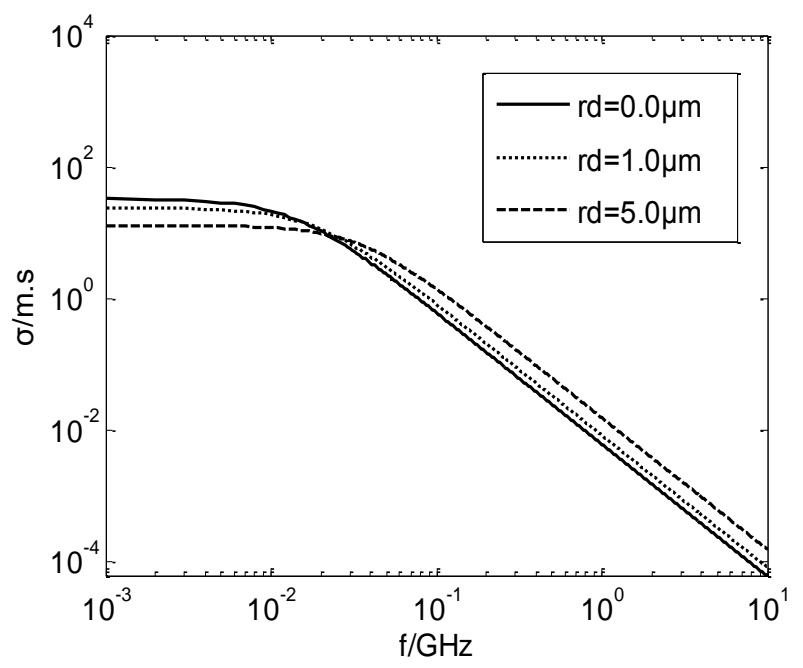

Fig. 1 The effect of dust radius on the conductivity

Higher dust density, in addition to dust radius effect, will also increase the collisions between electron and charged dust particle. As a reference case, we set the $r_{d}=1.0 \mu \mathrm{m}, n_{d}=10^{12} \mathrm{~m}^{-3}$ and $n_{d}=10^{13} \mathrm{~m}^{-3}$, respectively, and the other parameters the same as Fig. 1. It can see from the Fig. 2 that the change trend is qualitatively the same as that in Fig.1.

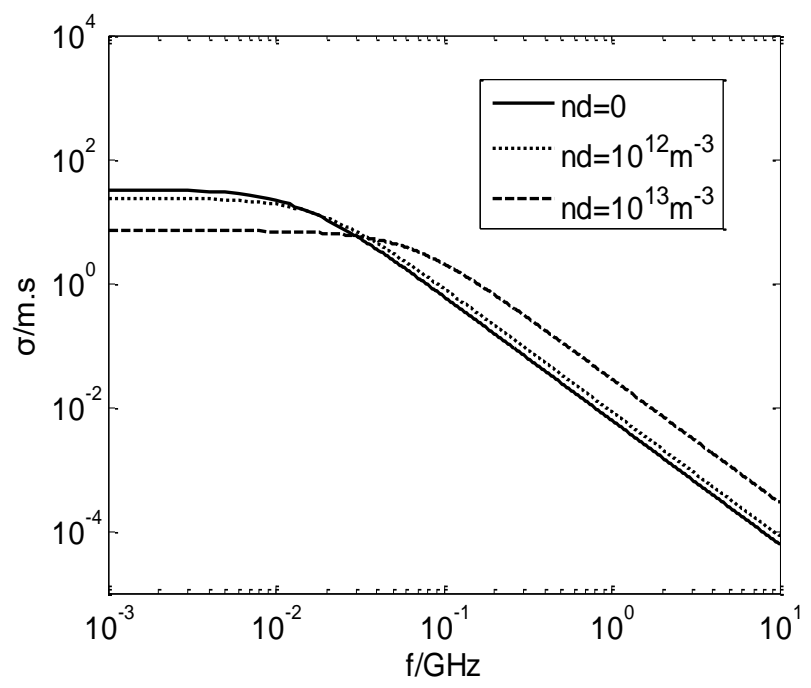

Fig. 2 The effect of dust density on the conductivity 
The dust particles are considered as mono-sized in the above analysis. In general case, the dust consists of different sizes. In order to investigate the effect of dust size distribution on the electrical conductivity, as a simple reference case, we introduce the power law distribution, which has been widely accepted in space plasmas. The differential form of the power law distribution is given by

$$
n_{d}(r) d r=k r^{-\beta} d r
$$

Where $k$ is the normalization constant and $\beta$ is the power law index, the total number of all dust in a rang of $\left[r_{\min }, r_{\max }\right]$ can be written as

$$
N_{t o t}=\int_{r_{\min }}^{r_{\max }} n_{d}(r) d r=\frac{k}{1-\beta}\left(r_{\max }^{1-\beta}-r_{\min }^{1-\beta}\right)
$$

For the mono-sized dust particle, all the particle have the same size, which equals to the mean radius, the corresponding mean radius can be given as follows:

$$
\bar{r}=\frac{\int r n_{d}(r) d r}{\int n_{d}(r) d r}=\frac{1-\beta}{2-\beta}\left(\frac{r_{\max }^{2-\beta}-r_{\min }^{2-\beta}}{r_{\max }^{1-\beta}-r_{\min }^{1-\beta}}\right)
$$

We define that $\lambda=r_{\text {max }} / r_{\text {min }}$ and $\eta(\lambda, \beta)=v_{e d} / \bar{v}_{e d}$ are the ratio of electron-dust collision for power law size distribution to the mono-sized with the size of average power law distribution. We choose the above typical parameters and examine the effects of this size distribution on the collision rate between the electron and charged dust particle. Fig.3 shows the variation of $\eta$ with respect to $\lambda$ for different $\beta=4$, 5, respectively. It can be concluded from Fig.3 that the value of $\eta$ turns out to be larger than 1, which means that electron-dust collision rate for power law distribution are larger than that of mono-sized case with average dust size. We can also see that, for smaller $\beta$, the $\eta$ can reaches relative higher values and increases as $\lambda$ increases. However, for larger $\beta$, the influence of $\lambda$ on the result may be neglected, when $\lambda \geq 10$. 


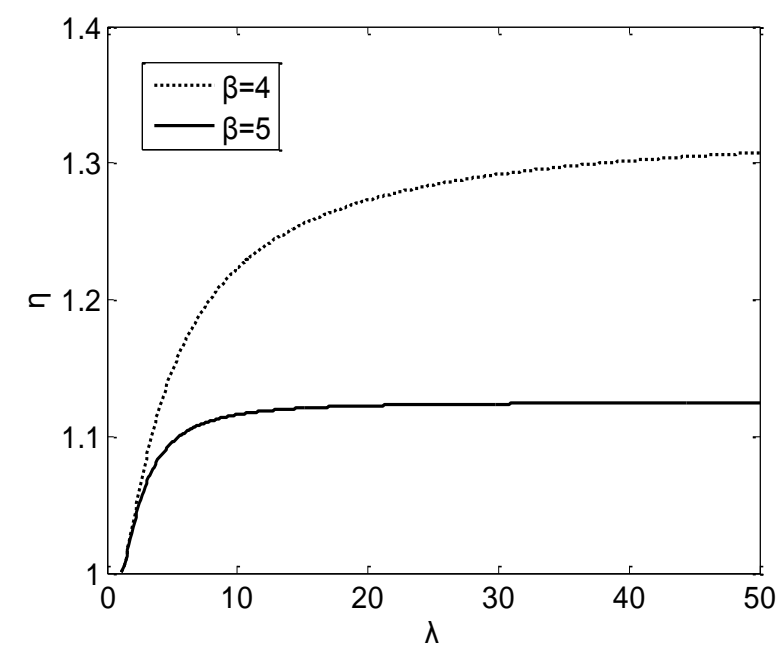

Fig.3 the variation of $\eta$ with respect to $\lambda$ for different $\beta$

\section{Conclusion}

In this paper, we have investigated the conductivity of dusty plasma by taking into account the collisions of electrons with charged dust particle. We have shown that the presence of charged dust particle can significantly alter the conductivity of plasma. For the low frequency, the additional collision provided by charged dust particles can reduce the conductivity. However, when the electromagnetic wave greater than the collision frequency, the additional collision increases the conductivity of plasma. All of these effects are depends on dust radius, density as well as their charge numbers on the surface. The obtained results will support an enhanced understanding of the electromagnetic wave propagation processes in some dusty plasma. Such as the additional absorption of microwave signal pass through a solid rocket exhaust plume.

Furthermore, considering that real dusty plasma consists of dusts of different sizes, the influence of size distribution is also examined for a power law distribution. It is shown that the electron-dust collision rate for a power law distribution exceeds the rate for mono-sized dust size. This means that compare to uniform size, the power law size distribution have greater influences on the conductivity. It is also interesting to extend this model to dusty plasma by considering other dust size distribution.

It should be noted that charge numbers on dust surface has been assumed as constant in the present study. It has been confirmed that the charges on dust surface are subject to fluctuation 20,21 . Thus, for low frequency waves, particularly when 
frequency is close to the dust charge fluctuation frequency, this process should also be considered in the analysis of conductivity. Furthermore, the dust in plasma not only has various sizes, but also consists of various shapes as well as dielectric material. For example, the dust particles are often typically elongated or rotating form. In this case, the dust can acquire a rotational motion due to their interaction with surrounding particle. The angular frequency of such rotation often can reach a rather large value and can also modify the electromagnetic property of plasma. Meanwhile, if the dust particles are dielectric material, the own property of the material may also contribution to the conductivity. All of these effects on the conductivity are worth further investigation.

\section{Acknowledgements}

The authors wish to express their sincere thanks to Dr. Jia Jieshu for her some constructive comments. This work was supported by the National Science Foundation of China (NSFC) under grant 40831062 and 61205093.

[1] U. De Angelis, R. Bingham and V. N. Tsytovich, J. Plasma Phys 42 (1989) 445.

[2] R. L. Merlino, A. Barkan, C. Thompson and N. D’ Angelo, Phys. Plasmas, 5 (1998), 1607.

[3] V. Fortov, A. Ivlev, S. Khrapak, A. Khrapak and G. Morfill, Physics Reports 421 (2005), 1.

[4] S. Khrapak and G. Morfill, Contrib. Plasma Phys. 49 (2009) 148.

[5] S. V. Vladimirov and K. Ostrikov, Phys. Rep. 393 (2004) 175-380.

[6] V. N. Tsytovich, G. E. Morfill, and H. Thomas, Plasma Phys. Rep. 28 (2002) 623.

[7] G. E. Morfill, V. N. Tsytovich, and H. Thomas, Plasma Phys. Rep. 29 (2003) 1.

[8] H. Thomas, G. E. Morfill, and V. N. Tsytovich, Plasma Phys. Rep. 29 (2003) 895.

[9] M. S. Sodha, Shikha Misra and S. K. Mishra, Phys. Plasmas 17 (2010) 113705.

[10] M. Lampe, G. Joyce, G. Ganguli and V. Gavrishchaka, Phys. Plasmas 7 (2000) 3851.

[11] P. K. Shukla and A. A. Mamun, Introduction to dusty plasma physics, Institute Physics Publishing, (2002).

[12] A. F. Alexandrov, L. S. Bogdankevich, and A. A. Rukhadze, Principles of Plasma Electrodynamics, (1984). 
[13] S. A. Khrapak and G. Morfill, Phys. Plasma 8 (2001) 2649.

[14] S. A. Khrapak, Alexey V. lvlev and Gregor E. Morfill, Physical Review E 70 (2004) 056405.

[15] S. A. Khrapak and Gregor E. Morfill, Physical Review E 69 (2004) 066411.

[16] M. Rosenberg and G. Sorasio, J. Spacecraft Rockets 43 (2006) 245.

[17] Y. V. Platov, S. A. Chernouss, and M. J. Kosch, J. Spacecraft Rockets 41 (2004) 667.

[18] T. Aba, K. Fujita K. H. Ogawa and I. Funaki, $31^{\text {th }}$ AIAA Plasma dynamics and Lasers Conference 19-22 Denver, Colorado (2000).

[19] Peter Meuris, Planet. Space Sci., 45(9), 1171-1174, 1997.

[20] Jin-Xiu Ma, P. K. Shukla and M. Y. Yu, Phys. Lett. A 198 (1995) 357.

[21] R. K. Varma, Phys. Plasmas 7 (2000) 3505. 